Classification

Physics Abstracts

47.20

\title{
Large scale instability of nonlinear standing waves
}

\author{
P. Coullet (*) \\ Laboratoire de Physique de la Matière Condensée, Parc Valrose, 06034 Nice, France
}

S. Fauve

Groupe de Physique des Solides de l'Ecole Normale Supérieure, 24 rue Lhomond, 75005 Paris, France

and E. Tirapegui (**)

Faculté des Sciences, Université Libre de Bruxelles, CP 226, 1050 Bruxelles, Belgium

(Reçu le 7 mai 1985, accepté le 8 juillet 1985)

\begin{abstract}
Résumé. - Nous établissons les équations aux dérivées partielles non linéaires qui gouvernent la stabilité à grande échelle d'une structure cellulaire unidimensionnelle oscillante, apparaissant dans un système hors équilibre, invariant par translations d'espace et de temps, et par réflexion d'espace. Nous montrons l'existence d'une instabilité oscillatoire, conduisant à un régime quasipériodique possédant deux échelles spatiales distinctes.
\end{abstract}

\begin{abstract}
We present the nonlinear phase equations describing the stability of a time-periodic onedimensional spatial pattern, that arises in a system which is invariant by space and time translations and space reflection symmetry. We show that a large scale oscillatory instability can occur, leading to a quasiperiodic temporal regime with two different spatial scales.
\end{abstract}

A problem of great current interest is the stability of patterns which arise in many nonlinear dissipative systems driven far from equilibrium by an external forcing homogeneous in time and space. Time periodic chemical reactions, convection rolls or Taylor cells are well known examples of such temporal or spatial patterns. Long wavelength modes are very general features of these dissipative structures, and can be described by the slowly varying temporal or spatial phase of the periodic pattern. The concept of phase dynamics has been successfully used by several authors in order to study pattern dynamics in reaction diffusion equations [1, 2], in the Rayleigh-Bénard instability [3] and in the Couette-Taylor instability [4]. In this Letter we study the stability of an oscillatory spatial pattern that occurs through a supercritical bifurcation. Corresponding situa-

(*) Also Observatoire de Nice, BP 139, 06003 Nice, France.

$\left({ }^{* *}\right)$ Also Universidad de Chile, Casilla 5487, Santiago, Chile. 
tions exist for Couette flow between counter-rotating cylinders [5], or for thermal convection in the presence of a salinity gradient [6]. We show that two phases, $\Theta$ and $\Sigma$, associated to the broken translational invariances in time and space, are necessary to describe the long wavelength instabilities of the oscillatory pattern; we derive nonlinear equations for $\Theta$ and $\Sigma$, and show that the oscillatory pattern itself can undergo a bifurcation that leads to a quasiperiodic state.

We consider a one space dimensional physical system described by a set of scalar fields (more general situations could be considered) : $\mathbb{U}(x, t)=\left(U_{1}(x, t), U_{2}(x, t), \ldots, U_{N}(x, t)\right)$ which is invariant by space reflection $x \rightarrow-x$, and by constant space and time displacements $x \rightarrow x+\Sigma, t \rightarrow t+\Theta$. The possibility of observing in such a system a standing wave is related to the existence of modes of finite spatial period, $\Phi_{k}(x)=\Phi_{k}$ exp $i k x$, undergoing a bifurcation at $k=k_{\mathrm{c}}$ with an imaginary growth rate $i \omega$, through variation of an external constraint $\mu$. Let

$$
\eta_{k}=\mu-\xi_{0}\left(k^{2}-k_{\mathrm{c}}^{2}\right)^{2}+\mathcal{O}\left(k^{2}-k_{\mathrm{c}}^{2}\right)^{3}+i\left[\omega+\omega_{1}\left(k^{2}-k_{\mathrm{c}}^{2}\right)+\mathcal{O}\left(k^{2}-k_{\mathrm{c}}^{2}\right)^{2}\right]
$$

be the growth rate of the critical modes. The instability occurs at $\mu=0$ and for $\mu>0$ one expects a behaviour characterized by rapid space and time scales $\frac{2 \pi}{k_{\mathrm{c}}}$ and $\frac{2 \pi}{\omega}$ respectively. One looks for $\mathbb{U}$ in the form

$$
\mathbb{U}(x, t)=A(X, T) \exp i\left(\omega t-k_{\mathrm{c}} x\right) \Phi_{k_{\mathrm{c}}}+B(X, T) \exp i\left(\omega t+k_{\mathrm{c}} x\right) \Phi_{k_{\mathrm{c}}}+\mathcal{U}
$$

where $U$ stands for linear and nonlinear corrections which are assumed to be small for $\mu \ll 1$, and $A(X, T), B(X, T)$ are the two slowly varying complex fields necessary to describe the onset of the instability. In equation(2) we have represented by $X$ and $T$ the slow space and time variables. The fields $A$ and $B$ describe respectively the amplitudes of waves propagating to the right and to the left, and obey amplitude equations which can be derived by standard asymptotic methods [7].

$$
\begin{aligned}
& \partial_{T} A=F\left(A, B, \bar{A}, \bar{B} ; \partial_{X}\right) \\
& \partial_{T} B=G\left(A, B, \bar{A}, \bar{B} ; \partial_{X}\right)
\end{aligned}
$$

where $\bar{A}$ and $\bar{B}$ stand for the complex conjugates of $A$ and $B$. The symmetries of the system determine in fact the form of these equations [8] since they imply the invariance of equations (3) under the transformations

$$
\begin{gathered}
A \rightarrow A \exp -i \Sigma, B \rightarrow B \exp i \Sigma \\
A \rightarrow A \exp i \Theta, B \rightarrow B \exp i \Theta \\
X \rightarrow-X, A \rightarrow B, B \rightarrow A
\end{gathered}
$$

and this leads at lowest order to the equations

$$
\begin{aligned}
& A_{T}+A_{X}=\mu A+(1+i \alpha) A_{X X}-(\varepsilon+i \beta)|A|^{2} A-(\gamma+i \delta)|B|^{2} A \\
& B_{T}-B_{X}=\mu B+(1+i \alpha) B_{X X}-(\gamma+i \delta)|A|^{2} B-(\varepsilon+i \beta)|B|^{2} B
\end{aligned}
$$

where we have simplified the coefficients by appropriate scalings, and $\varepsilon= \pm 1$. We note that although symmetry arguments allow $(1+i \eta) A_{X}$ and $( \pm 1+i \alpha) A_{X X}$, stability requirements lead to (5). From now we take equations (5) as a model and we assume we are in the supercritical situation : $\varepsilon=1$ and $\gamma>-1$. The spatially homogeneous solutions

$$
A_{0}(T)=Q_{A} \exp i \Omega_{A} T, B_{0}(T)=Q_{B} \exp i \Omega_{B} T,
$$

describe either : 
a) propagating waves corresponding to

$$
Q_{A}^{2}=0 ; \quad Q_{B}^{2}=\mu, \Omega_{B}=-\beta \mu
$$

or

$$
Q_{A}^{2}=\mu, \Omega_{A}=-\beta \mu ; \quad Q_{B}^{2}=0
$$

which are stable with respect to spatially homogeneous perturbations when $\gamma>1$,

b) standing waves which correspond to

$$
Q_{A}^{2}=Q_{B}^{2}=\frac{\mu}{(1+\gamma)}, \Omega_{A}=\Omega_{B}=-\frac{(\beta+\delta)}{(1+\gamma)} \mu
$$

which are stable when $-1<\gamma<1$. We will consider this last situation in this Letter [9].

In order to study the stability of this solution with respect to inhomogeneous perturbations it is useful to remark that equations (5) admit a more general class of solutions

$$
\begin{aligned}
& A_{0}(X, T)=Q_{A} \exp \left(-i p X+i \Omega_{A} T\right) \\
& B_{0}(X, T)=Q_{B} \exp \left(i q X+i \Omega_{B} T\right)
\end{aligned}
$$

with

$$
\begin{gathered}
Q_{A}^{2}=\frac{\mu(1-\gamma)-p^{2}+\gamma q^{2}}{1-\gamma^{2}} \\
Q_{B}^{2}=\frac{\mu(1-\gamma)+\gamma p^{2}-q^{2}}{1-\gamma^{2}} \\
\Omega_{A}=-\mu \frac{\delta+\beta}{1+\gamma}+p-\left(\alpha+\frac{\delta \gamma-\beta}{1-\gamma^{2}}\right) p^{2}+\frac{\delta-\beta \gamma}{1-\gamma^{2}} q^{2} \\
\Omega_{B}=-\mu \frac{\delta+\beta}{1+\gamma}+q+\frac{\delta-\beta \gamma}{1-\gamma^{2}} p^{2}-\left(\alpha+\frac{\delta \gamma-\beta}{1-\gamma^{2}}\right) q^{2}
\end{gathered}
$$

The stability of this solution is studied considering the dynamics of an arbitrary perturbation

$$
\begin{aligned}
A(X, T) & =A_{0}(X, T)+\mathcal{A}(X, T) \exp \left(-i p X+i \Omega_{A} T\right) \\
B(X, T) & =B_{0}(X, T)+\mathcal{B}(X, T) \exp \left(i q X+i \Omega_{B} T\right)
\end{aligned}
$$

In the limit of homogeneous perturbations, i.e. $\mathcal{A}$ and $\mathfrak{B}$ independent of $X$, one find two types of linear modes. The former associated with the real parts of $\mathcal{A}$ and $\mathcal{B}$ are stable, at least for $p$ and $q$ sufficiently small, and correspond to amplitude perturbations. The latter, which are marginal, correspond to phase perturbations and are associated to the imaginary parts $\phi$ and $\psi$ of $\mathcal{A}$ and $\mathfrak{B}$. The elimination of the amplitude modes leads to the phase equations which are in all generality of the form

$$
\begin{array}{r}
\phi_{T}=a_{1} \phi_{X}+b_{1} \psi_{X}+a_{2} \phi_{X X}+b_{2} \psi_{X X}+\cdots+g \phi_{X}^{2}+h \phi_{X} \psi_{X}+k \psi_{X}^{2}+\cdots \\
\psi_{T}=-b_{1}^{\prime} \phi_{X}-a_{1}^{\prime} \psi_{X}+b_{2}^{\prime} \phi_{X X}+a_{2}^{\prime} \psi_{X X}+\cdots+k^{\prime} \phi_{X}^{2}+h^{\prime} \phi_{X} \psi_{X}+g^{\prime} \psi_{X}^{2}+\cdots
\end{array}
$$

where the coefficients are functions of $p$ and $q$. Equations (12) have the exact solutions

$$
\begin{aligned}
& \phi=-r X+\Omega_{\phi} T \\
& \psi=s X+\Omega_{\psi} T
\end{aligned}
$$


with

$$
\begin{aligned}
& \Omega_{\phi}=-a_{1} r+b_{1} s+g r^{2}-h r s+k s^{2} \\
& \Omega_{\psi}=b_{1}^{\prime} r-a_{1}^{\prime} s+k^{\prime} r^{2}-h^{\prime} r s+g^{\prime} s^{2}
\end{aligned}
$$

which can be interpreted in the limit $r \rightarrow 0, s \rightarrow 0$ as modifications of the wavenumbers of the initial solutions $: p \rightarrow p+r / Q_{A}, q \rightarrow q+s / Q_{B}$. Thus we have

$$
\begin{aligned}
& \Omega_{A}\left(p+r / Q_{A}, q+s / Q_{B}\right) \simeq \Omega_{A}(p, q)+\Omega_{\phi} \\
& \Omega_{B}\left(p+r / Q_{A}, q+s / Q_{B}\right) \simeq \Omega_{B}(p, q)+\Omega_{\psi} .
\end{aligned}
$$

In order to compute the coefficients $\left(a_{1}, a_{1}^{\prime}, b_{1}, b_{1}^{\prime}, g, h, l, g^{\prime}, h^{\prime}, l^{\prime}\right)$ we expand the left hand side of (15) in $r$ and $s$ and identify the terms in $r, s, r^{2}, r s$ and $s^{2}$. In the case $p=q=0$, i.e. when one considers the homogeneous solution (8), the phase equations become

$$
\begin{aligned}
& \phi_{T}=-\phi_{X}+a_{2} \phi_{X X}+b_{2} \psi_{X X}+\cdots+g \phi_{X}^{2}+h \phi_{X} \psi_{X}+k \psi_{X}^{2}+\cdots \\
& \psi_{T}=\psi_{X}+b_{2} \phi_{X X}+a_{2} \psi_{X X}+\cdots+k \phi_{X}^{2}+h \phi_{X} \psi_{X}+g \psi_{X}^{2}+\cdots
\end{aligned}
$$

with

$$
\begin{gathered}
g=\left.\frac{1}{2 Q_{A}} \frac{\partial^{2} \Omega_{A}}{\partial p^{2}}\right|_{p=q=0}=-\sqrt{\frac{1+\gamma}{\mu}}\left(\alpha+\frac{\delta \gamma-\beta}{1-\gamma^{2}}\right) \\
h=\left.\frac{-1}{Q_{B}} \frac{\partial^{2} \Omega_{A}}{\partial p \partial q}\right|_{p=q=0}=0 \\
k=\left.\frac{Q_{A}}{2 Q_{B}^{2}} \frac{\partial^{2} \Omega_{B}}{\partial q^{2}}\right|_{p=q=0}=\sqrt{\frac{1+\gamma}{\mu}}\left(\frac{\delta-\beta \gamma}{1-\gamma^{2}}\right) \\
a_{2}=1+\frac{\alpha \beta}{1-\gamma^{2}}-\frac{\alpha \gamma \delta}{1-\gamma^{2}} \\
b_{2}=\alpha \frac{\delta-\beta \gamma}{1-\gamma^{2}} .
\end{gathered}
$$

Equations (16) are invariant by $X \rightarrow-X, \phi \rightarrow \psi, \psi \rightarrow \phi$, which reflects the space reflection invariance of the initial system which is not broken by the choice of the homogeneous solution (8). The stability of this solution is studied considering first the linear version of(16). The eigenvalues of the corresponding linear operator are given by

$$
s_{ \pm}(k)= \pm i k+s_{2} k^{2}+\mathcal{O}\left(k^{3}\right)
$$

where

$$
s_{2}=-1-\alpha \frac{\beta-\gamma \delta}{1-\gamma^{2}}
$$

A bifurcation occurs when $s_{2}$ changes sign and it represents the appearance of a low frequency which modulates the standing waves envelope. This bifurcation occurs at zero spatial wavenumber if the coefficient $s_{4}$ of $k^{4}$ in (18) is positive. In fact $s_{2}$ and $s_{4}$ can vanish simultaneously [10]. This defines a codimension-two surface in parameter space in the vicinity of which the bifurcation can occur first either at zero or finite spatial frequency.

It is immediate to interpret the phases $\phi$ and $\psi$ in terms of the spatial and temporal phases, 
$\Sigma=\frac{\psi-\phi}{2}, \Theta=\frac{\psi+\phi}{2}$, of the oscillating pattern. In these new variables equations

become

$$
\begin{aligned}
& \Theta_{T}=\Sigma_{X}+a \Theta_{X X}+\cdots+u \Theta_{X}^{2}+u \Sigma_{X}^{2}+\cdots \\
& \Sigma_{T}=\Theta_{X}+b \Sigma_{X X}+\cdots+w \Theta_{X} \Sigma_{X}+\cdots
\end{aligned}
$$

These equations describe in all generality the phase stability of a time periodic pattern with respect to long wavelength perturbations, independently of the bifurcation problem considered in this Letter. The only hypothesis is the reflection invariance of the considered structure which implies the invariance of (20) under the transformation

$$
X \rightarrow-X, \Theta \rightarrow \Theta, \quad \Sigma \rightarrow-\Sigma .
$$

These equations appear naturally when the problem can be reduced to the coupling of scalar and pseudo-scalar phases [4].

Let us note that the case $\gamma>1$ corresponds to nonlinear wave propagation, the phase instability of which has already been studied [2]. The case $\gamma=1$ is particular since at the considered order the amplitude equations admit a one-parameter family of solutions which continuously connects the purely propagating wave solution (7) to the standing wave solution(8). A new phase $\chi$ is associated with this symmetry. Although higher order terms generally break it, it can have observable consequences in the neighbourhood of the instability onset.

Finally in the case $-1<\gamma<1$, we have shown that the standing waves can undergo a bifurcation leading to a quasiperiodic temporal regime. This oscillatory instability occurs either at zero spatial frequency, as usual for phase instabilities, or at finite spatial frequency. Detailed calculations together with an analysis of the various phase equations, and their numerical simulations are in preparation.

\section{Acknowledgments.}

This work has been partly supported by the CNRS (ATP « Dynamique des fluides géophysiques et astrophysiques ") and the CPAI.

\section{References}

[1] Howard, L. N. and Kopell, N., Studies in Applied Math. 56 (1977) 95-145.

[2] Kuramoto, Y., Prog. Theor. Phys. 71 (1984) 1182-1196.

[3] Pomeau, Y. and Manneville, P., J. Physique Lett. 40 (1979) L-609-612.

[4] Brand, H. and Cross, M. C., Phys. Rev. A 27 (1983) 1237-1239.

Brand, H., Prog. Theor. Phys. 71 (1984) 1096-1099.

TABeling, P., J. Physique Lett. 44 (1983) L-665-672.

[5] Di Prima, R. C. and Grannick, R. N., IUTAM Svmp. on Instability of Continuous Systems, H. Leipholz ed. (Springer) 1971.

[6] Bretherton, C. S., Woods Hole Oceanographic Institution Technical Report, (1981) 81-102 ;

Bretherton, C. S. and Spiegel, E. A., Phys. Lett. A 96 (1983) 152-156.

[7] See for instance Newell, A. C., Lectures in Applied Math. 15 (1974) 157-163 and references therein.

[8] Analogous symmetry arguments have been used to derive amplitude equations for bifurcations in the Couette-Taylor problem; see Chossat, P. and Iooss, G., preprint Université de Nice (1984).

[9] These different cases can occur for the instability of the Couette flow between counter-rotating cylinders ; see DemaY, Y. and Iooss, G., to be published in J. Méc. (1985).

[10] The coefficients $s_{n}$ have been calculated up to $n=6$ with the help of MACSYMA. We have

$$
s_{4}=\frac{-\alpha \mu\left(\gamma^{4}-2 \beta \delta \gamma^{3}+3 \beta^{2} \gamma^{2}+3 \delta^{2} \gamma^{2}-2 \gamma^{2}-6 \beta \delta \gamma+\beta^{2}+\delta^{2}+1\right)+2 \alpha \gamma(\beta \gamma-\delta)}{2\left(1-\gamma^{2}\right)^{3} \mu^{2}} .
$$

